\title{
The effects of Tai Ji Quan training on limits of stability in older adults
}

This article was published in the following Dove Press journal:

Clinical Interventions in Aging

4 August 2014

Number of times this article has been viewed

\author{
Fuzhong $\mathrm{Li}^{1,2}$ \\ 'Oregon Research Institute, \\ Eugene, OR, USA; ${ }^{2}$ Shanghai University \\ of Sport, Shanghai, People's Republic \\ of China
}

Background: Limits of stability, defined as the ability to maintain the center of gravity within the boundary of the base of support, is critically important for older adults in performing their activities of daily living. However, few exercise programs specifically tailored to enhance limits of stability exist. The primary purpose of this study was to determine whether a therapeutically designed intervention, Tai Ji Quan: Moving for Better Balance (TJQMBB), could improve limits of stability in older adults. A secondary purpose was to examine concomitant change in limits of stability and physical performance as a result of the intervention.

Methods: A single-group design was used in which 145 community-dwelling older adults (average age: 75 years) were enrolled in TJQMBB classes, participating twice weekly for 48 weeks. Primary outcome measures were three indicators of limits of stability (LOS) (endpoint excursion, movement velocity, and directional control), with secondary measures of physical performance being Timed Up and Go and 50-foot speed walk (in seconds), which were assessed at baseline, 24 weeks, and 48 weeks. Changes in the repeated measures of outcome variables were analyzed via latent curve analysis.

Results: At 48 weeks, a significant rate of change (improvement) over time was observed in the three limits of stability indicators (endpoint execution: $8.30 \%$ LOS, $P<0.001$; movement velocity: 0.86 degrees/second, $P<0.001$; directional control: $6.79 \%$ of $100, P<0.001$ ); all reached a threshold of real change as judged by the minimal detectable change values. Improvements in the three limits of stability measures were concomitantly correlated with improved (reduced times) performance scores in the Timed Up and Go $(-0.30,-0.45$, and -0.55 , respectively) and 50 -foot walk $(-0.33,-0.49$, and -0.41 , respectively).

Conclusion: In this single-group study, community-dwelling older adults trained through TJQMBB significantly improved their limits of stability, providing preliminary support for the use of TJQMBB as a therapeutic modality for enhancing functional activities in older adults.

Keywords: mobility, balance, activities of daily living, exercise, elderly

\section{Introduction}

In older adults, performance of many habitual daily activities and tasks requires moving and controlling the center of gravity (defined as the vertical projection of the center of mass) to various positions within the base of support. However, effective displacement of the center of gravity during either self-initiated voluntary movements or externally triggered perturbations requires the capacity to remain within the limits of stability, which is described as the maximum distance a person can intentionally displace his or her center of gravity from a midline vertical position in any direction without losing balance and stepping or grasping for support. ${ }^{1-4}$

Thus, a constricted limits of stability boundary or uncontrolled center of gravity movement can impair functional balance of older adults and constrain safe performance of their daily activities such as reaching, bending, walking, gait initiation,
Key Laboratory of Exercise and Health Sciences, Shanghai University of Sport, No 650, Qing Yuan Huan Road, Yangpu District, Shanghai 200438, People's Republic of China Email fuzhongl@gmail.com; fuzhongl@ori.org 
and moving from sit-to-stand. ${ }^{5}$ Current literature suggests that aging is associated with diminished limits of stability capacity $^{6-9}$ due to multiple factors, including reduced ankle muscle strength ${ }^{10}$ and poor cutaneous mechanoreceptor function in the soles of the feet, thus exposing older adults to more instability in functional daily activities and a higher risk of falling. ${ }^{11,12}$

Therefore, from the perspectives of injury prevention and/or clinical rehabilitation of physical function, developing exercise intervention strategies that maximize the center of gravity sway envelope (ie, the area an individual can move his or her center of gravity with control within the base of support $)^{4}$ and subsequently expand the limits of stability boundary becomes functionally important for improving balance and mobility and, critically, for reducing the risk of falling among the elderly, which is a significant public health problem worldwide. ${ }^{13,14}$

Because of its functional significance and implications for risk of falling, limits of stability should be integrated into exercise balance training programs that target fall prevention in older adults. However, among the many types of group- and evidence-based exercise programs $\mathrm{s}^{15-18}$ designed to prevent falls, few have a specific, tailored focus on integrating training elements that would maximize limits of stability. Given that constrained limits of stability can lead to an increased propensity to fall in older adults, ${ }^{1,19}$ fall prevention programs that explicitly integrate therapeutic balance training of limits of stability are needed.

Taking a therapeutic approach, Li et $\mathrm{al}^{20,21}$ developed the Tai Ji Quan: Moving for Better Balance (TJQMBB) program that specifically includes an element of training on limits of stability. Integrating Tai Ji Yin-Yang theory ${ }^{22}$ and theories of motor control and clinical practice, , ${ }^{1,23}$ TJQMBB uses an innovative strategy of embracing a complementary interplay between stability and instability (dualities of YinYang) during training to achieve balance equilibrium. ${ }^{20}$ More specifically, training focuses on eliciting both reactive and anticipatory responses to self-induced, deliberately controlled and coordinated Tai Ji Quan movements aimed at stimulating postural adjustments to progressively increase the sway envelope.

The primary purpose of this study, therefore, was to determine whether a year-long training intervention with TJQMBB, given its specific focus on training limits of sway, could enhance limits of stability among communitydwelling older adults. It was hypothesized that TJQMBB would enhance indicators of limits of stability. In light of the functional significance of the limits of stability, a secondary purpose was to examine concomitant changes between limits of stability and physical performance resulting from the intervention.

\section{Methods}

\section{Study design}

The study design involved a single-group pre- and postintervention with repeated measures, conducted between May 2012 and January 2014.

\section{Participants and procedures}

Participants in this study were a subset of communitydwelling older adults recruited for a large community-based dissemination project through promotions (newsletters, flyers, sign-up sheets) at local senior centers in Lane County, OR, USA. The research protocol was approved by the Institutional Review Board of Oregon Research Institute, and an informed consent was obtained from all participants.

Those who met the following criteria were eligible for participation: (1) 65 years of age or over; (2) physically mobile (objectively assessed by the ability to walk at least one block with or without an assistive device); (3) showing no severe cognitive deficits, as defined by the Mini-Mental State Evaluation ( $\geq 23) ;{ }^{24}$ and (4) having medical clearance from a health care provider. Individuals who responded to the promotions were subsequently contacted and scheduled for an in-office visit where the details of the study were explained. Following a consent process, baseline data were collected at a research laboratory prior to the beginning of the TJQMBB program with follow-ups at 24 and 48 weeks. Trained research staff collected demographic and study outcome data.

\section{Intervention}

The TJQMBB program, with the conceptual basis and technical detail fully described elsewhere, ${ }^{20}$ is a balance training modality based on applications of Tai Ji Quan with a specific focus on training limits of stability and sensory integration. Eligible participants participated in a full 60-minute TJQMBB class session taught by a trained instructor twice per week for 48 consecutive weeks. In terms of the training protocol, each session began with Tai Ji Quan-based movement warm-up exercises (5-10 minutes), followed by teaching and practicing the core components (described in the following section) (40-45 minutes), and ending with a simple set of breathing exercises (3-5 minutes).

The training protocol involves a core set of adapted "Yang Style"22 Tai Ji Quan-based forms with built-in 
practice variations and a subset of mini therapeutic movements aimed at stimulating and integrating musculoskeletal and sensory systems via performance of self-initiated Tai Ji Quan movements. Exercise sessions were structured from an easy-to-difficult learning-and-practice progression with an emphasis on within-session practice integration of forms, variations of the forms, and mini movements. Both seated and standing exercises were implemented, with seated exercises emphasizing weight shift, trunk rotation, transitioning from sit-to-stand and stand-to-sit, and standing exercises focusing on the features described in the following section.

During each session, participants practiced choreographed Tai Ji Quan forms/movements (eg, Part Wild Horse's Mane) while engaging in controlled circular ankle sways (ie, lateral, anterior-posterior, medial-lateral together) that moved the center of gravity progressively toward and near the edge of the base of support with planted feet; trunk-driven rotational (laterally and posteriorly) weight shift; plantar/ dorsiflexion during transitioning between moves; reactive and proactive (anticipatory) postural adjustments in response to self-induced perturbed actions on either "fixed support" or "change-in-support", 25 and active and coordinated eyehead-hand movements.

Throughout the training and practice, emphasis was placed on (1) an interplay between stability (static movements) and instability (self-induced movement perturbations) in performing each form/move; (2) functional ankle-based training by mimicking reaching, tilting, stepping, and turning tasks; and (3) a variable approach that stressed practice variations in movement speeds, range of joint motion, movement directions and patterns, and base of foot support.

\section{Outcome measures}

The primary outcome was limits of stability, which consisted of three indicators: (1) endpoint excursion; (2) movement velocity; and (3) directional control, assessed by computerized dynamic posturography (Balance Master ${ }^{\circledR}$ System; NeuroCom ${ }^{\circledR}$, Clackamas, OR, USA). Endpoint excursion assesses the limits of self-initiated movements as participants shift or lean their center of gravity toward the theoretical limit (100\%) in eight prespecified target directions without loss of balance (scores expressed as a percentage; \% LOS). Movement velocity assesses the average speed of the center of gravity movement from the center to the eight targets (scores expressed as degrees/second). Directional control provides a measure of movement accuracy by comparing the amount of movement toward the target to the amount of extraneous movement (scores expressed as \% of 100).
Higher values in all three indicators indicate better limits of stability. Reliability of limits of stability has been established ${ }^{26}$ or shown in previous studies. ${ }^{21,27}$ In this study, 24-week test-retest reliability (correlation) ranged from 0.52-0.64 ( 0.57 for endpoint excursion, 0.52 for movement velocity, and 0.64 for directional control).

Secondary measures included physical performance measures of the: (1) Timed Up and Go test ${ }^{28}$ and (2) 50-foot speed walk, adopted from the Physical Performance Test. ${ }^{29}$ Timed Up and Go measured the time (in seconds) taken to rise from a chair, walk 10 feet $(3 \mathrm{~m})$, return, and sit down, with a lower time indicating better mobility. The 50 -foot speed walk measured the time (in seconds) it took participants to walk as quickly as possible 25 feet out and 25 feet back. The 6-month test-retest reliability was 0.82 for Timed Up and Go and 0.91 for the 50 -foot walk test, respectively.

\section{Data analysis}

Intervention outcomes were analyzed on a sample size of 145 participants who provided at least two data points (baseline, 24-week, and/or 48-week follow-up). Incomplete data were handled through the last observation carried forward method. Descriptive statistics (mean, standard deviation) were performed to describe the study sample characteristics. Coefficient of variation was calculated for each variable across time as the ratio of standard deviation to the mean, multiplied by 100 . Minimal detectable change (MDC) scores were calculated for each of the limits of stability indicators to determine whether the change score was at or above the minimal level of detectible change. MDC is based on the standard error of measurement and calculated at a $95 \%$ confidence interval $\left(\mathrm{MDC}_{95}\right)$ using the formula of:

$$
\text { Standard error of measurement } \times \sqrt{ } \mathrm{n} \times 1.96 \text {, }
$$

where the standard error of measurement is derived from the square root of the mean square error term in the repeated measures analysis of variance and $\sqrt{ } \mathrm{n}$ is the number of measurements used.

Intervention effect on the primary and secondary outcomes was analyzed via latent growth curve analysis. In the latent curve analysis, the primary interest was on the following estimates: (1) slope means on each of three limits of stability indicators and (2) a slope-to-slope correlation between each of the limits of stability indicators and the two physical performance measures. Given the single-group intervention design, change at 48 weeks from baseline was estimated for the indicators of endpoint excursion and directional control 
using the pooled estimates from prior studies. ${ }^{21,27}$ Assuming an alpha level of 0.05 with $80 \%$ power to detect $7 \%$ LOS points difference in endpoint excursion and $8 \%$ of 100 points in directional control with an anticipated dropout rate of $30 \%$ for the 48 -week intervention, a minimal sample size of 70 participants for the TJQMBB was planned.

All statistical tests were two-sided, and $P<0.05$ was considered statistically significant. All descriptive analyses including MDC scores were performed using SPSS version 17 (SPSS Inc., Chicago, IL, USA) and Microsoft ${ }^{\oplus}$ Office Excel $^{\circledR}$ software for Windows ${ }^{\circledast}$ (Microsoft Corporation, Redmond, WA, USA). Changes in the latent means of the limits of stability indicators and correlations of changes between limits of stability and physical performance measures were performed using Mplus software (v 6; Muthén \& Muthén, Los Angeles, CA, USA). ${ }^{30}$

\section{Results}

From April 2012 to January 2014, 169 older adults were screened. Of these, 151 (89\%) were eligible for participation. The major reasons for being determined ineligible were age $(n=13)$, time conflict $(n=3)$, or significant change in health $(n=2)$. In addition, six eligible participants $(4 \%)$ were removed because they attended only one session and provided no follow-up data. Thus, the analytic sample size in the study was 145 .

\section{Baseline characteristics}

Study population characteristics at baseline are presented in Table 1. The mean age at enrollment was approximately 75 years, $79 \%$ of the participants were women, and approximately $92 \%$ were Caucasian. In all, 124 participants (85\%) reported having two or more chronic conditions, 70 (48\%) were taking two or more prescribed medications, and $73(50 \%)$ reported having fallen at least once in the previous 6 months, with about $25 \%$ of those having fallen two or more times. A total of 98 participants (68\%) reported having some level of fear of falling.

\section{Intervention attrition and adherence}

Of the total 145 participants, 21 (14\%) dropped out of the study during the 48 -week intervention period, due primarily to reasons such as deterioration in medical conditions $(\mathrm{n}=14)$, change in health status $(n=5)$, or relocation $(n=2)$. There were, however, no significant differences in baseline demographics and functional characteristics between the participants who completed the intervention and those who dropped out of the study (completers: $\mathrm{n}=124$ versus dropouts: $\mathrm{n}=21$ ).
Table I Baseline characteristics of participants

\begin{tabular}{|c|c|}
\hline \multirow[t]{2}{*}{ Demographics } & \multirow{2}{*}{$\frac{\mathrm{N}=145}{\text { Estimate }}$} \\
\hline & \\
\hline Age, mean (standard deviation), years & $75(7)$ \\
\hline Female sex, n (\%) & II 5 (79) \\
\hline White, n (\%) & $133(92)$ \\
\hline \multicolumn{2}{|l|}{ Education attainment, $\mathrm{n}(\%)$} \\
\hline High school diploma or lower & $43(30)$ \\
\hline College degree or higher & $102(70)$ \\
\hline \multicolumn{2}{|l|}{ Chronic disease conditions, $\mathrm{n}(\%)$} \\
\hline 0 & $7(5)$ \\
\hline 1 & $14(10)$ \\
\hline$\geq 2$ & $124(85)$ \\
\hline \multicolumn{2}{|l|}{ Number of medications taking, $\mathrm{n}(\%)$} \\
\hline 0 & $26(18)$ \\
\hline 1 & $34(24)$ \\
\hline 2 & $36(25)$ \\
\hline$\geq 3$ & $34(33)$ \\
\hline \multicolumn{2}{|l|}{ Health status, n (\%) } \\
\hline Excellent/very good & $60(4 I)$ \\
\hline Good/fair & $84(58)$ \\
\hline Poor/very poor & $\mathrm{I}(\mathrm{I})$ \\
\hline \multicolumn{2}{|l|}{ Falls in past 6 months, $\mathrm{n}(\%)$} \\
\hline 0 & $72(49)$ \\
\hline 1 & $37(26)$ \\
\hline 2 & $21(15)$ \\
\hline 3 & $9(6)$ \\
\hline$\geq 4$ & $6(4)$ \\
\hline \multicolumn{2}{|l|}{ Afraid of falling, $n(\%)$} \\
\hline No & $47(32)$ \\
\hline Somewhat & $56(39)$ \\
\hline Quite a lot/very much & $42(29)$ \\
\hline
\end{tabular}

The average number of class sessions attended per participant for the intervention (a total of 96 sessions) was 66 sessions (range: 5-96 sessions; median: 73 sessions) for the whole sample ( $\mathrm{N}=145$, including dropouts $[\mathrm{n}=21]$ ) compared to 73 sessions (range: $28-96$ sessions; median: 78 sessions) for those who completed the 48 -week intervention $(n=124)$. The percentage attendance for the intervention was $69 \%$ (median: $76 \%$ ) for the whole sample $(\mathrm{N}=145)$ and $76 \%$ (median: $81 \%$ ) for the completers $(n=124)$.

\section{Change in primary and secondary outcomes}

Table 2 provides descriptive statistics (mean, standard deviation) for the three primary and two secondary outcome variables across the three measurement time points. The observed means for each of the three limits of stability show a linear improvement over time. Change from baseline values were $17.21 \% \operatorname{LOS}(32 \%)$ for endpoint execution, 1.71 degrees/second (68\%) for movement velocity, and 
Table 2 Descriptive statistics in the measures of limits of stability and physical performance outcomes over time (48 weeks)

\begin{tabular}{|c|c|c|c|}
\hline \multirow[t]{2}{*}{ Outcomes } & \multicolumn{3}{|l|}{$N=\mid 45$} \\
\hline & Baseline & 24 weeks & 48 weeks \\
\hline \multicolumn{4}{|l|}{ Limits of stability } \\
\hline Endpoint excursion, \% limits of stability & $53.80 \pm 8.92(16.58)$ & $64.59 \pm 8.48(13.13)$ & $71.10 \pm 8.89(12.50)$ \\
\hline Movement velocity, degrees/second & $2.5 I \pm 0.77(30.68)$ & $3.37 \pm 0.74(21.95)$ & $4.22 \pm 0.91(21.56)$ \\
\hline Directional control, \% of 100 & $65.45 \pm 8.16(12.47)$ & $74.78 \pm 8.16(10.91)$ & $77.86 \pm 8.02(10.30)$ \\
\hline Timed Up and Go (seconds) & $10.72 \pm 1.76(16.42)$ & $9.22 \pm 1.86(20.17)$ & $8.54 \pm 1.65(19.32)$ \\
\hline 50-foot speed walk (seconds) & $14.99 \pm 3.76(25.08)$ & $13.80 \pm 3.24(23.48)$ & $12.0 \mid \pm 3.09(25.73)$ \\
\hline
\end{tabular}

Note: The data represent mean \pm standard deviation (coefficient of variation).

$12.41 \%$ of $100(19 \%)$ for directional control compared to $\mathrm{MDC}_{95}$ values, which were $16.88 \%$ LOS for endpoint excursion, 1.63 degrees/second for movement velocity, and $12.14 \%$ of 100 for directional control, respectively. Results of the latent growth analysis are presented in Table 3, which further indicates that there was a significant rate of change (as reflected in latent linear slope means) in the indicators of endpoint excursion, movement velocity, and directional control over the 48 -week intervention period.

\section{Correlations in change between primary and secondary outcomes}

The analyses also show a significant small-to-moderate negative correlation (indicating improvement) between rate of change in each of the limits of stability indicators and the two physical performance measures over time (Table 3).

\section{Intervention safety}

There were no significant adverse events attributable to the intervention observed during the intervention period. Three falls were reported during warm-up sessions, but the participants sustained no injuries and continued the classes.

\section{Discussion}

This is the very first study to evaluate the effect of a specifically tailored Tai Ji Quan training program on improving limits of stability, an important prerequisite for performing many of the functional activities of daily living in older adults. The results showed that 48 weeks of TJQMBB training significantly improved older adult limits of stability as reflected in increases in the indicators of movement excursion, velocity, and control, and that increased limits of stability were concomitantly associated with improved physical performance. Thus, with no significant adverse events during the study period, it appears that the intervention is an appropriate one for this at-risk population.

While cross-sectional studies that showed that older adult practitioners of Tai Ji Quan tended to exhibit better stability limits compared to nonpractitioners ${ }^{31,32}$ provided early nonintervention data on the potential of Tai Ji Quan to enhance limits of stability, more recent intervention studies from the author's group that integrated limits of sway training demonstrated the benefit of this focused Tai Ji Quan program for improving limits of stability in older adults and patients with Parkinson's disease..$^{21,27}$ In contrast, other Tai Ji Quan-based studies, ranging from 4 to 12 months of intervention, ${ }^{33,34}$ have not shown significant improvements in postural stability measures in older adults. The TJQMBB program differs from the programs in these other studies in a clinically important way in that this approach transforms traditional martial arts-based training into a therapeutically oriented training paradigm that specifically emphasizes the practice of deliberate, voluntarily controlled Tai Ji Quan

Table 3 Change in the study outcome variables over time and correlations between limits of stability and physical performance measures

\begin{tabular}{|c|c|c|c|c|}
\hline \multirow[t]{2}{*}{ Variables } & \multicolumn{4}{|l|}{$N=145$} \\
\hline & $\begin{array}{l}\text { Baseline } \\
\left.\text { (intercept }^{\mathrm{a}}\right)\end{array}$ & $\begin{array}{l}\text { Rate of } \\
\left.\text { change (slope }{ }^{b}\right)\end{array}$ & $\begin{array}{l}\text { Correlation with } \\
\text { Timed Up and } G_{0}{ }^{c}\end{array}$ & $\begin{array}{l}\text { Correlation with } \\
50 \text {-foot speed walk }\end{array}$ \\
\hline Endpoint excursion, \% limits of stability & $54.53 *$ & $8.30 *$ & $-0.30^{*}$ & $-0.33^{*}$ \\
\hline Movement velocity, degrees/second & $2.51 *$ & $0.86 *$ & $-0.45^{*}$ & $-0.49 *$ \\
\hline Directional control, \% of 100 & $66.61 *$ & $6.79 *$ & $-0.55^{*}$ & $-0.4 I^{*}$ \\
\hline
\end{tabular}

Notes: $* \mathrm{P}<0.00 \mathrm{I}$; athe value represents the average at baseline; ${ }^{b}$ the value represents linear change over time; ${ }^{c}$ these coefficients represent correlations between latent variables with measurement error removed. 
postural actions. These actions include manipulation of the center of gravity movements, variations in the base of support, and adaptation training with reactive and proactive actions to self-induced perturbations, with the purpose of increasing the sway envelope and thereby expanding the limits of stability, with the ultimate goal of reducing the risk of falling.

Although there are currently no guidelines for gauging clinically meaningful change in the measures of limits of stability, this 48-week, twice-per-week training schedule intervention appears to have provided a substantive change over time in the measures used in this study: $32 \%$ on endpoint excursion, $68 \%$ on movement velocity, and $19 \%$ on directional control. In addition, MDC was demonstrated in all three outcomes after the intervention. Improvements in these limits of stability indicators support the potential for the TJQMBB program to address problems of improper weight shifts that are observed in elderly people residing in longterm care. ${ }^{35}$ Consistent with findings from a cross-sectional study, ${ }^{5}$ the results demonstrate that improved measures on limits of stability through this Tai Ji Quan training in older adults was sufficiently sensitive to elicit change in clinical measures of balance and mobility, suggesting that training with TJQMBB offers the potential to improve functional activities and activities of daily living, including sit-to-stand, walking, turning, and stand-to-sit tasks.

The author's intervention approach linking martialarts-based Tai Ji Quan to clinical and functional training of balance in older adults offers some practical implications. From an applied perspective, the training approach used in this study can be readily introduced in various settings. For example, as an effective balance training modality, TJQMBB can be readily adopted in diverse communities for the purpose of preventing falls ${ }^{36,37}$ and in health care settings to assist patients with balance disorders and fall risk problems. ${ }^{21}$ Uniquely different from all other existing Tai Ji Quan-based programs, TJQMBB also has a specific, tailored clinical focus in that training incorporates therapeutic approaches such as closed and open kinetic chain movements $;{ }^{38}$ motor control mechanisms through manipulation of movement magnitude, speed, direction, and base of support; and training of functional activities that take into account interactions between individuals, tasks at hand, and environmental constraints imposed on the tasks. ${ }^{23}$ These specific training features thus make the program amenable and applicable to clinical training and rehabilitation that address neuromuscular and movement disorders in patients with balance and gait problems and who are at high risk of falls. The clinical relevance of the program, therefore, is apparent.

The mechanism by which the training protocol improves the limits of stability indicators in this study was not explored and therefore remains unclear. Limits of stability is known to be influenced by a number of physiological and biomechanical factors, ${ }^{4,23}$ including lower extremity strength, range of ankle motion, and cutaneous mechanoreceptors in the feet, ${ }^{2}$ acting either alone or in combination. Through integration of Tai Ji Quan and physical therapy, the protocol in this study puts great emphasis on controlled weight shifting and ankle movement sway, alternation between a narrow stance and a wide stance to continually change the base of support, rotational trunk-driven movements, and dorsiflexion and plantar flexion during movement transitioning. These therapeutically tailored training features may have made an impact on important sensorimotor elements that contribute to enhanced limits of stability. Therefore, it is plausible that improvement in the limits of stability performance may be attributed to unmeasured improvements in flexion/extension ankle torques, the range of ankle motion, movement recovery strategies, or sensory integration - features that are inherent in TJQMBB training. Some of these mechanisms of action may be revealed by evaluating the kinematics of body movement or conducting kinetic analysis of these ankle swing-based static and dynamic movement exercises, thus offering a better understanding of the movement strategies and forces contributing to the intervention-induced change in limits of stability.

This study has some limitations. Its design does not include a control condition, making it difficult to draw a firm conclusion regarding the true intervention effect of TJQMBB on limits of stability. Therefore, the results should be corroborated by future clinical randomized controlled trials. Given the importance of plantarflexor muscle strength in limits of stability, ${ }^{10}$ another limitation is the lack of a measure of ankle muscle strength, which precludes the opportunity to examine the role of ankle strength in the observed improved limits of stability outcomes. Finally, the study used theoretical limits of stability values derived from computerized dynamic posturography that correspond to the maximum range in which center of gravity can be moved safely without changing the base of support. ${ }^{26}$ However, the capacity of stability limits may vary depending on the task performed; ${ }^{23}$ therefore, consideration of performance-based or functional limits of stability resembling real-world situations may be needed in future assessments. ${ }^{39}$ 


\section{Conclusion}

The TJQMBB program, a transformed protocol of traditional Tai Ji Quan training approaches, was found to improve limits of stability in older adults, and the improved limits of stability were associated with improved functional performance. Within the limitations of the current study, the combined results provide promising preliminary data that suggest the potential utility of TJQMBB for increasing limits of stability in older adults, thereby enhancing functional performance of habitual daily activities.

\section{Acknowledgment}

The work presented in this paper is supported by a research grant from the National Institute on Aging (R01-AG034956).

\section{Disclosure}

The author reports no conflict of interest in this work.

\section{References}

1. Horak FB, Shumway-Cook A. Clinical implications of postural control research. In: Duncan PW, editor. Balance: Proceedings of The APTA Forum, Nashville, TN, USA, June 13-15, 1989. Alexandria, VA: American Physical Therapy Association; 1990:105-111.

2. Horak FB. Postural orientation and equilibrium: what do we need to know about neural control of balance to prevent falls? Age Ageing. 2006;35(Suppl 2):ii7-ii11.

3. McCollum G, Leen TK. Form and exploration of mechanical stability limits in erect stance. J Mot Behav. 1989;21(3):225-244.

4. Nashner LM. Practical biomechanics and physiology of balance. In: Jacobson GP, Newman CW, Kartush JM, editors. Handbook of Balance of Function Test. Clifton Park, NY: Thomson Delmar Learning; 1997:261-279.

5. Topp R, Mikesky A, Thompson K. Determinants of four functional tasks among older adults: an exploratory regression analysis. J Orthop Sports Phys Ther. 1998;27(2):144-153.

6. Hageman PA, Leibowitz JM, Blanke D. Age and gender effects on postural control measures. Arch Phys Med Rehabil. 1995;76(10):961-965.

7. King MB, Judge JO, Wolfson L. Functional base of support decreases with age. J Gerontol. 1994;49(6):M258-M263.

8. Holbein-Jenny MA, McDermott K, Shaw C, Demchak J. Validity of functional stability limits as a measure of balance in adults aged 23-73 years. Ergonomics. 2007;50(5):631-646.

9. Tsang WW, Hui-Chan CWY. Effects of exercise on joint sense and balance in elderly men: tai chi versus golf. Med Sci Sports Exerc. 2004;36(4):658-667.

10. Melzer I, Benjuya N, Kaplanski J, Alexander N. Association between ankle muscle strength and limit of stability in older adults. Age Ageing. 2009;38(1):119-123.

11. Girardi M, Konrad HR, Amin M, Hughes LF. Predicting fall risks in an elderly population: computer dynamic posturography versus electronystagmography test results. Laryngoscope. 2001;111(9):1528-1532.

12. Trueblood PR, Hodson-Chennault N, McCubbin A, Youngclarke D. Performance and impairment-based assessments among community dwelling elderly: sensitivity and specificity. J Geriatr Phys Ther. 2001;24(1):2-6.

13. Falls among older adults: an overview [webpage on the Internet]. Atlanta, GA: Centers for Disease Control and Prevention; 2013 [updated September 20, 2013]. Available from: http://www.cdc.gov/Homeand RecreationalSafety/Falls/adultfalls.html. Accessed January 5, 2014.
14. World Health Organization. WHO Global Report on Falls Prevention in Older Age. Geneva: World Health Organization; 2007. Available from: http://www.who.int/ageing/publications/Falls_prevention7March.pdf. Accessed January 5, 2014.

15. Barnett A, Smith B, Lord SR, Williams M, Baumand A. Communitybased group exercise improves balance and reduces falls in at-risk older people: a randomized controlled trial. Age Ageing. 2003;32(4): 407-414.

16. Freiberger E, Menz HB, Abu-Omar K, Rutten A. Preventing falls in physically active community-dwelling older people: a comparison of two intervention techniques. Gerontology. 2007;53(5):298-305.

17. Lord SR, Castell S, Corcoran J, et al. The effect of group exercise on physical functioning and falls in frail older people living in retirement villages: a randomized, controlled trial. J Am Geriatr Soc. 2003;51(12):1685-1692.

18. Wolf SL, Barnhart HX, Kutner NG, McNeely E, Coogler C, Xu T. Reducing frailty and falls in older persons: an investigation of tai chi and computerized balance training. J Am Geriatr Soc. 1996;44(5): 489-497.

19. Duncan PW, Weiner DK, Chandler J, Studenski S. Functional reach: a new clinical measure of balance. J Gerontol. 1990;45(6):M192-M197.

20. Li F. Transforming traditional Tai Ji Quan techniques into integrative movement therapy - Tai Ji Quan: Moving For Better Balance. Journal of Sport and Health Science. 2013;3(1):9-15.

21. Li F, Harmer P, Stock R, et al. Implementing an evidence-based fall prevention program in an outpatient clinical setting. J Am Geriatr Soc. 2013; 61(12):2142-2149.

22. Committee of Chinese Wushu Encyclopedia. Chinese Wushu Encyclopedia. Beijing: Encyclopedia of China Publishing House; 1998.

23. Shumway-Cook A, Woollacott MH. Motor Control: Translating Research into Clinical Practice. Baltimore, MD: Lippincott Williams \& Wilkins; 2012.

24. Folstein MF, Folstein SE, McHugh PR. "Mini-mental state". A practical method for grading the cognitive state of patients for the clinician. J Psychiatrics Res. 1975;12(3):189-198.

25. Maki BE, Mcllroy WE. The role of limb movements in maintaining upright stance: the "change-in-support" strategy. Phys Ther. 1997;77(5): 488-507.

26. NeuroCom. Balance Manager Systems: Clinical Operations Guide. Clackamas, OR: NeuroCom; 2008.

27. Li F, Harmer P, Fitzgerald K, et al. Tai chi and postural stability in patients with Parkinson's disease. N Engl J Med. 2012;366(6):511-519.

28. Podsiadlo D, Richardson S. The Timed "Up and Go": a test of basic functional mobility for frail elderly persons. J Am Geriatr Soc. 1991; 39(2):142-148.

29. Brown M, Sinacore DR, Binder EF, Kohrt WM. Physical and performance measures for the identification of mild to moderate frailty. J Gerontol A Biol Sci Med Sci. 2000;55(6):M350-M355.

30. Muthén LK, Muthén BO. Mplus User's Guide. 6th edition. Los Angeles, CA: Muthén \& Muthén; 1998.

31. Tsang WW, Hui-Chan CW. Effects of tai chi on joint proprioception and stability limits in elderly subjects. Med Sci Sports Exerc. 2003; 35(12):1962-1971.

32. Gyllensten AL, Hui-Chan CW, Tsang WW. Stability limits, single-leg jump, and body awareness in older tai chi practitioners. Arch Phys Med Rehabil. 2010;91(2):215-220.

33. Wolf SL, Barnhart HX, Ellison GL, Coogler CE. The effect of tai chi quan and computerized balance training on postural stability in older subjects. Phys Ther. 1997;77(4):371-381

34. Woo J, Hong A, Lau E, Lynn H. A randomised controlled trial of tai chi and resistance exercise on bone health, muscle strength, and balance in community-living elderly people. Age Aging. 2007;36(3): 262-268.

35. Robinovitch SN, Feldman F, Yang Y, et al. Video capture of the circumstances of falls in elderly people residing in long-term care: an observational study. Lancet. 2013;381(9860):47-54. 
36. Fink D, Houston K. Implementing an evidence-based Tai Ji Quan program in a multicultural setting: a pilot dissemination project. Journal of Sport and Health Science. 2014;3(1):27-31.

37. Leung J. Implementing Tai Ji Quan: Moving for Better Balance in realworld settings: success and challenges. Journal of Sport and Health Science. 2014;3(1):34-35.
38. Ellenbecker TS, Davies GJ. Closed Kinetic Chain Exercise: A Comprehensive Guide To Multiple-Joint Exercises. Champaign, IL: Human Kinetics; 2001.

39. Jbabdi M, Boissy P, Hamel M. Assessing control of postural stability in community-living older adults using performance-based limits of stability. BMC Geriatr. 2008;8:8.

\section{Publish your work in this journal}

Clinical Interventions in Aging is an international, peer-reviewed journal focusing on evidence-based reports on the value or lack thereof of treatments intended to prevent or delay the onset of maladaptive correlates of aging in human beings. This journal is indexed on PubMed Central, MedLine,
CAS, Scopus and the Elsevier Bibliographic databases. The manuscript management system is completely online and includes a very quick and fair peer-review system, which is all easy to use. Visit http://www.dovepress. com/testimonials.php to read real quotes from published authors. 